\title{
Theory and Methology
}

\section{Joint replacement in an operational planning phase}

\author{
R. Dekker, R.E. Wildeman * , R. van Egmond \\ Econometric Institute, Erasmus University Rotterdam, P.O. Box 1738, 3000 DR Rotterdam, Netherlands
}

Received November 1993; revised December 1994

\begin{abstract}
We consider the problem of combining replacements of multiple components in an operational planning phase. Within an infinite or finite time horizon, decisions concerning replacement of components are made at discrete time epochs. The optimal solution of this problem is limited to only a small number of components. We present a heuristic rolling horizon approach that decomposes the problem; at each decision epoch an initial plan is made that addresses components separately, and subsequently a deviation from this plan is allowed to enable joint replacement. This approach provides insight into why certain actions are taken. The time needed to determine an action at a certain epoch is only quadratic in the number of components. After dealing with harmonisation and horizon effects, our approach yields average costs less than $1 \%$ above the minimum value.
\end{abstract}

Keywords: Maintenance; Planning; Replacement; Markov decision programming; Dynamic programming

\section{Introduction}

Maintenance activities often require the same preparatory work. Consequently, joint execution of such maintenance activities can save costs. For instance, if a maintenance job has to be carried out at an unmanned installation, simultaneous execution of maintenance activities can save travel costs; if maintenance implies shutdown of a production system, then down-time costs can be saved when carrying out maintenance activities at the same time. In this paper we investigate a multicomponent replacement problem in an operational planning phase. At discrete time epochs of an infinite or finite time horizon decisions whether or not to replace a component or a

\footnotetext{
${ }^{*}$ Corresponding author.
}

group of components have to be made, so as to minimise the average or total costs (we do not consider discounted costs in this paper).

For a recent literature overview of the field of maintenance of multi-component systems, we refer to the review article by Cho and Parlar [2]. Methods that are published so far for modelling maintenance of multi-component systems are not tractable for a large number of components, unless a very special structure is assumed.

The maintenance of a deteriorating system is frequently described using Markov decision theory (see, e.g., Howard [9], who was the first to use such a problem formulation). However, since the state space in such problems grows exponentially with the number of components, the Markov decision modelling of multi-component systems is not tractable (see, e.g., Bäckert and Rippin [1], and Haurie and L'Ecuyer [8]). This implies that 
for many components heuristic methods have to be applied. For instance, Dekker and Roelvink [3] present a heuristic replacement criterion in case always a fixed group of components is replaced.

In the Markov decision modelling of multicomponent maintenance, often opportunistic control-limit strategies are used: mandatory actions are induced by upper limits, which creates opportunities for other actions that are allowed if lower limits are reached. For example, an $(n, N)$-strategy for two identical components prescribes to replace a component when it has failed or when its age has reached the value $N$ and, if one of the components is replaced, to replace the other when its age is greater than or equal to $n$ $(<N)$. Van der Duyn Schouten and Vanneste [11] study $(n, N)$-strategies, but provide an algorithm for only two identical components. Wijnmalen and Hontelez [12] determine control-limit rules for a model with inspection and repair. These authors propose an iterative procedure based on decomposition and aggregation, but only few computational results are presented. A problem with these strategies (and with Markov decision models in general) is that it is mostly not known beforehand what actions are going to be taken. This planning aspect is important when, e.g., work preparation is necessary. Besides, strategies of this type do not yield much insight into the question why certain actions are combined: The only information they provide are the optimal control parameters and the average costs. Neither do they allow operational planning in which short-term information can be incorporated.

Wildeman, Dekker and Smit [14] propose a general approach for the combination of maintenance activities in an operational planning phase for block-replacement-like models. In this paper we extend this approach to Markov decision problems. To this end we decompose and plan the Markov decision chain that describes the evolution of the whole system. Planning is possible by taking expectations and by assuming a most likely action at each decision epoch in the case of no premature failures. (Another possibility, frequently used by engineers, is to use resid- ual lifetimes as states in the Markov chain. See, for instance, Worm and Van Harten [15].) Decomposition is applied in the sense that at each decision epoch an initial plan is made that addresses components separately. Subsequently, we adapt these plans to enable joint execution of maintenance, thus benefitting from savings in setup work. These ideas are worked out in a rolling horizon approach.

The advantage of our approach is that it provides insight into the combination process, rather than only providing optimal control limits. Furthermore, it allows planning, and as joint replacements are decided on a short-term basis, operational information such as one-off savings can also be taken into account. Another important advantage is that the multi-component Markov decision model is decomposed into one-component models that can easily be solved.

In our approach we use a combination technique and a dynamic programming algorithm of Wildeman, Dekker and Smit [14]. The combination technique originates from Dekker, Smit and Losekoot [4], however they formulate a set partitioning problem that may be far too large to solve. Furthermore, both papers consider problems of the block-replacement type in a continuous-time setting, and their actions are not being influenced by failures. We consider age-replacement strategies, in discrete time, where failure maintenance can be combined with preventive maintenance. Using the dynamic programming algorithm of Wildeman, Dekker and Smit [14] has the advantage that the computational effort of determining an action at a certain decision epoch is only quadratic in the number of components. Hence we can handle many components, that need not be identical.

Our approach can also be applied to other maintenance systems (see Wildeman, Dekker and Smit [14]) and to other Markov decision models (e.g., inspection models). Furthermore, the approach can be applied in production control and inventory control areas (see Dekker and Wildeman [5]), since the same problem structure applies there.

Wildeman, Dekker and Smit [14] present a general approach but do not study its perfor- 
mance. In this paper we compare our approach with optimal strategies, that can only be determined in specific cases with a small number of components $(\leqslant 4)$. We also compare our approach with $(n, N)$-strategies of Van der Duyn Schouten and Vanneste [11]. Numerical experiments are carried out to see how our approach performs under different circumstances; we explain what negative effects play a role in the performance and how these effects can be dealt with.

The structure of this paper is as follows. Section 2 provides a mathematical formulation of the problem. In Section 3 we present our heuristic rolling horizon approach. Results of a numerical investigation into the performance of this approach are given in Section 4. In Section 5 we conclude with a short evaluation.

\section{Problem formulation}

Consider a system with $n$ independently operating components $1, \ldots, n$. Each component is subject to stochastic failure, and has a known time-to-failure distribution. The system is considered at discrete time epochs, which will be called decision epochs. For ease of analysis it is assumed that these epochs are equidistant, but this is not essential. Consequently, one time unit corresponds to one period (the time between two epochs).

If at a decision epoch component $i$ is still working, it can be replaced preventively against costs $r_{i}$. If component $i$ turns out to have failed during the last period, it is immediately replaced correctively against costs $r_{i}+b_{i}$, where $b_{i}$ are breakdown costs. The time needed for replacement is negligible. After a component is replaced, a new identical component starts with age equal to zero. Preventive and corrective replacement of different components can be combined. We assume that $r_{i}$ includes set-up costs $\Delta c$ that are the same for all components. This implies that combining replacement of $m$ components (being preventive or corrective) yields a cost reduction of $(m-1) \Delta c$. (This is not an uncommon assumption, as set-up costs are due to, e.g., crew travel- ling, scaffolding, shutdown, etc., which are assumed to be the same for all components. Another practical motivation is that it is very hard to obtain more specific data; no present-day management information system supports a data structure for each possible combination of components.)

A component is said to be found in state $j$ at a decision epoch when it has not failed and its age equals $j$ periods. Denote by $q_{i}^{j}$ the probability that component $i$ fails during the next period, given that its age equals $j$ at the beginning of this period, and define $p_{i}^{j}=1-q_{i}^{j}$. The lifetime distributions are supposed to have finite support, i.e., when component $i$ has reached its maximal lifelength $m_{i}$, it certainly will fail during the next period. When component $i$ has failed we say that it is in state $m_{i}+1$.

The system described above is considered for an infinite and a finite time horizon. For an infinite horizon the objective is the minimisation of the long-term average costs, for a finite horizon the minimisation of the total costs over the horizon. (If costs are discounted, then the objective could be the minimisation of the total discounted costs in both cases. However, we do not consider discounted costs in this paper.) The system is modelled as a Markov decision process. A state in this process is determined by the combination of the states of all components. As each component $i$ has $m_{i}+1$ possible states and there are $n$ components, the state space consists of $\prod_{i=1}^{n} m_{i}+1$ states. Thus the dimension equals the number of components.

\section{Rolling horizon approach}

In this section we give a heuristic rolling horizon approach to the problem of joint replacement of multiple components of the system described in the previous section. Firstly, in Section 3.1, we give a motivation of the approach. In Section 3.2 a general description is given. Subsequently, in Sections 3.3 and 3.4 we distinguish between an infinite and a finite time horizon. Finally, in Section 3.5 we discuss the heuristic elements of our approach. 


\subsection{Motivation}

Since the state space of the Markov decision process defined in Section 2 grows exponentially with the number of components, the model can be solved to optimality for only a few components. To allow many components, we apply in our approach a decomposition by considering components separately. For each component an individual control limit is defined. If $x_{i}^{*}$ is the individual control limit of component $i$, then this control-limit strategy prescribes to replace component $i$ when its age equals or exceeds $x_{i}^{*}$. A control-limit strategy follows from a one-component replacement model with breakdown costs $b_{i}$ and replacement costs $r_{i}$, for an infinite time horizon, thereby ignoring savings from joint execution of maintenance. By applying standard arguments from renewal theory, it can be shown (see, for instance, Van der Duyn Schouten and Vanneste [11]) that $x_{i}^{*}$ is the minimising argument of

$g_{i}(x):=\frac{r_{i}+b_{i}\left(1-\prod_{l=0}^{x-1} p_{i}^{l}\right)}{1+\sum_{j=2}^{x} \prod_{l=0}^{j-2} p_{i}^{l}}$,

where the empty sum equals zero and the empty product equals one.

In a following phase, savings from joint execution of maintenance are taken into account. These savings, initially ignored by considering components separately, can now be incorporated on an operational planning basis. The question is how this can best be done. Consider, for instance, two components 1 and 2, with $x_{1}^{*}=x_{2}^{*}=7$, and with ages 7 and 5, respectively. As the age of component 1 equals its control limit, replacement is due today. Though replacement of component 2 is not yet due, it can be cost-effective, viz. when the costs of advancement (replacing it today at age 5 instead of at its control limit) are less than the cost reduction obtained. The costs of advancement can be expressed in the relative values of components 1 and 2.

Corresponding with the control-limit strategy of component $i$ are the relative values $v_{i}^{j}$. These values, commonly used in Markov decision theory, can be interpreted as the relative costs (relative to the average costs $\left.g_{i}{ }^{*}=g_{i}\left(x_{i}^{*}\right)\right)$ of component $i$ being in state $j$ rather than in another state, and are generally given by the optimality equations

$v_{i}^{j}=\min _{a}\left\{c_{i}^{j}(a)+\sum_{k} p_{i}^{j k}(a) v_{i}^{k}\right\}-g_{i}^{*}$

(see, e.g. Tijms [10]). Here $a$ is the action taken, $c_{i}^{j}(a)$ are the costs of taking action $a$ when component $i$ is in state $j$, and $p_{i}^{j k}(a)$ is the probability of transition of component $i$ from state $j$ into state $k$ when action $a$ is chosen. It is not difficult to derive from the optimality equations the following expression for the values $v_{i}^{j}$ under the individual control-limit strategy of component $i$ :

$v_{i}^{j}= \begin{cases}p_{i}^{j} v_{i}^{j+1}+q_{i}^{j} v_{i}^{m_{i}+1}-g_{i}^{*}, & j=0, \ldots, x_{i}^{*}-1, \\ r_{i}+v_{i}^{0}, & j=x_{i}^{*}, \ldots, m_{i}, \\ b_{i}+r_{i}+v_{i}^{0}, & j=m_{i}+1 .\end{cases}$

Now it is easy to calculate the costs of advancing replacement of component 2. Replacement today, at age 5 instead of at age 7, costs $r_{2}$ and brings component 2 into state zero, where the relative value $v_{2}^{0}$ is paid. As otherwise $v_{2}^{5}$ would be paid, the extra costs are $r_{2}+v_{2}^{0}-v_{2}^{5}$. Combining replacement of components 1 and 2 yields a set-up cost reduction of $\Delta c$, so it is cost-effective to replace component 2 today if

$\Delta c>r_{2}+v_{2}^{0}-v_{2}^{5}$.

A question that arises next, is whether today is the best time to replace the combination of components 1 and 2 .

To answer this question, we first consider for each component $i$ an initially planned replacement epoch $t_{i}$. We take the most likely replacement epoch of component $i$, viz. the epoch at which it reaches its control limit if it will not fail before. A motivation for this choice is that when preventive replacement is cost-effective, the probability of a failure of component $i$ being of age $\leqslant x_{i}^{*}$ is small. (See, e.g., Geurts [7]; when the costs of a preventive-replacement strategy are at most $80 \%$ of the costs a failure-based strategy, 
then this probability is typically less than $30 \%$ ). Another motivation is that considering all possible failures of $n$ components leads to an exponential number of combinations, which is not tractable. Besides, there is no alternative when planning of replacement is required. Subsequently, we define for component $i$ a penalty function $h_{i}(\cdot)$, which expresses the costs of shifting replacement of component $i$ from the epoch $t_{i}$. With these penalty functions it is easy to determine the best replacement time of a combination. This is the time at which the sum of the penalty functions in the combination is minimal.

As the combination of components 1 and 2 is not the only combination possible, a following question is whether other combinations are better, for instance the combination of components 1,2 and 3 . To investigate this, we consider a finite planning horizon in which for each component its initially planned replacement epoch $t_{i}$ is considered. For this planning horizon a best combination structure, or grouping of components, is found. However, only a combination that is planned today is carried out. After that we start with a new planning horizon.

Altogether, we propose a rolling horizon approach that consists of five phases, which can be outlined as follows.

Phase 1: Individual control limits. Determine for each component $i$ an individual control limit $x_{i}^{*}$, which optimises the long-term average costs of the component.

Phase 2: Penalty functions. Derive for each component $i$ a penalty function $h_{i}(\cdot)$, expressing the costs of shifting replacement of component $i$ from its due time generated by its control limit $x_{i}^{*}$.

Phase 3: Individual planning. Determine at the current decision epoch for each component $i$ its first most likely replacement epoch $t_{i}$ according to its control limit $x_{i}^{*}$. The replacement epochs yield a finite planning horizon.

Phase 4: Joint replacement. Within this planning horizon the replacements are (optimally) combined, based on the penalty functions derived in Phase 2.

Phase 5: Rolling horizon step. Implement the combination that is planned at the current deci- sion epoch. At the following epoch a new state of the system is considered and the process is repeated from Phase 3. This is done for each decision epoch within the given infinite or finite time horizon.

\subsection{General description}

Below we specify the phases of our approach for the Markov decision replacement model.

\section{Phase 1: Individual control limits}

Individual control limits can be found with Eq (1). Efficient numerical methods, such as bisection, can be used to find the minimum. In our approach the control limits $x_{i}^{*}, i=1, \ldots, n$, will also be used when the problem is considered in a finite horizon, however, in that case they are not optimal.

Notice that the one-component control-limit strategy is a sort of discretised version of an age-replacement strategy.

\section{Phase 2: Penalty functions}

Let the replacement of component $i$ be initially and individually planned at decision epoch $t_{i}$. As will be explained in more detail in Phase 3, this $t_{\mathrm{i}}$, is the first epoch at which component $i$ has reached or exceeded its control limit $x_{i}^{*}$. The penalty function $h_{i}(\cdot)$ of component $i$ expresses the additional expected costs of shifting the replacement of component $i$ from this epoch $t_{i}$, which also effects all future replacements. The shift may be positive or negative (forward or backward in time, respectively).

In case at a decision epoch $t$ component $i$ turns out to have failed, it must be replaced immediately, i.e., no postponement is allowed. In that case the initially planned replacement epoch $t_{i}$ of component $i$ equals the current decision epoch: $t_{i}=t$, and a penalty function is defined that is infinite for each positive shift:

$h_{i}(\Delta t)=\infty$ for all $\Delta t>0$.

(Extending the model to allow a postponement of corrective replacement is obvious, but will not be done here.)

If component $i$ has not failed at its initially planned epoch $t_{i}$, postponing replacement will 
involve extra costs due to the probability that the component fails after the initially planned epoch, and it saves costs due to the fact that all future replacements are in expectation postponed as well. A similar argument holds for the case of a replacement being advanced. Now there are savings due to a decreased probability of failure, whereas we have to pay for the fact that all future replacements are in expectation advanced as well. For $\Delta t>0$, the value $h_{i}(\Delta t)$ expresses the expected costs of postponing replacement of component $i$ until epoch $t_{i}+\Delta t$ or until it fails, whichever happens first. For $\Delta t<0$, the value $h_{i}(\Delta t)$ expresses the expected costs of replacement of component $i$ at epoch $t_{i}+\Delta t$ instead of at epoch $t_{i}$ or at failure, whichever would have happened first. (Notice that, in the terminology of Wildeman, Dekker and Smit [14], we apply a long-term shift here.)

The penalty functions can be expressed in the relative values $v_{i}^{j}$ under the individual controllimit strategy of component $i$, see Eq. (2). Let $a_{i}$ be the age of component $i$ at the initially planned replacement epoch $t_{i}$, in case it will not fail. Take, for instance, $a_{i}=7$ and $\Delta t=2$. Consequently, the new replacement epoch will be $t_{i}+2$, if component $i$ will not fail before $t_{i}+1$. With probability $p_{i}^{7}$ component $i$ will be operating at epoch $t_{i}+1$, with probability $q_{i}^{7}$ it will not. In the first case it will be in state 8 , which is valued as $v_{i}^{8}$; in the latter it will be in state $m_{i}+1$, valued as $\iota_{i}^{m_{i}+1}$. As this transition takes one period, $g_{i}^{*}$ is saved in either case. In the first case (component $i$ is operating at epoch $t_{i}+1$ ), component $i$ will be replaced at epoch $t_{i}+2$. With probability $p_{i}^{7} p_{i}^{8}$ this replacement is preventive, which is valued as $v_{i}^{9}-g_{i}^{*}$; with probability $p_{i}^{7} q_{i}^{8}$ it is corrective, which is valued as $l_{i}^{m_{i}+1}-g_{i}{ }^{*}$. As otherwise state 7 is valued as $v_{i}^{7}$, we now have for $h_{i}(2)$ :

$$
\begin{aligned}
h_{i}(2)= & p_{i}^{7}\left(v_{i}^{8}-v_{i}^{7}-g_{i}^{*}\right)+q_{i}^{7}\left(v_{i}^{m_{i}+1}-v_{i}^{7}-g_{i}{ }^{*}\right) \\
& +p_{i}^{7} p_{i}^{8}\left(v_{i}^{9}-v_{i}^{8}-g_{i}^{*}\right) \\
& +p_{i}^{7} q_{i}^{8}\left(v_{i}^{m_{i}+1}-v_{i}^{8}-g_{i}{ }^{*}\right) .
\end{aligned}
$$

After substitution of the relative values (Eq. (2)) and after rewriting, we obtain the following expression:

$h_{i}(2)=\left(q_{i}^{7} b_{i}-g_{i}^{*}\right)+p_{i}^{7}\left(q_{i}^{8} b_{i}-g_{i}^{*}\right)$.
A general expression for $h_{i}(\Delta t)$ reads as follows:

$$
h_{i}(\Delta t)=\left\{\begin{array}{l}
\sum_{j=a_{i}}^{a_{i}+\Delta t-1}\left(q_{i}^{j} b_{i}-g_{i}^{*}\right) \prod_{l=a_{i}}^{j-1} p_{i}^{l} \\
\text { if } \Delta t \geqslant 0, \\
\sum_{j=a_{i}+\Delta t}^{a_{i}-1}\left(g_{i}^{*}-q_{i}^{j} b_{i}\right) \prod_{l=a_{i}+\Delta t}^{j-1} p_{i}^{l} \\
\text { if } \Delta t \leqslant 0,
\end{array}\right.
$$

with $a_{i}$ the age of component $i$ at the initially planned replacement epoch $t_{i}$, in case it will not fail. Here the convention is used that the empty sum equals zero and the empty product equals one. Further it should be noted that $\Delta t$ cannot be smaller than $-a_{i}$, since no replacement of a component can take place before it is operating, and that $\Delta t$ cannot be larger than $m_{i}+1-a_{i}$, since the maximal age is $m_{i}+1$. It is easily shown (see Dekker, Wildeman and Van Egmond [6]) that if $a_{i} \geqslant x_{i}^{*}$ and the probabilities $p_{i}^{j}$ are decreasing in $j$, the following holds with respect to the penalty functions: $h_{i}(\cdot)$ is strictly increasing for $\Delta t \geqslant 0$ and $h_{i}(\cdot)$ is strictly decreasing for $\Delta t \leqslant 0, h_{i}(\cdot) \geqslant 0$, and $h_{i}(0)=0$. In Phase 3 it follows from the definition of the individually planned replacement epoch $t_{i}$, that $a_{i} \geqslant x_{i}^{*}$.

\section{Phase 3: Individual planning}

Suppose the current decision epoch, at which an action has to be chosen, is epoch $t$. Denote by $t_{i}$ the individually planned replacement time (epoch) of component $i$. We take as individually planned time that epoch $(\geqslant t)$ at which the component reaches its control limit if it will not fail before. As replacements can be postponed, it may happen that the ages of some components have already exceeded their control limit at epoch $t$. In that case epoch $t$ automatically becomes the new individual replacement time of these components. The same holds for components that turn out to have failed at decision epoch $t$. Summarising, with age $_{i}$ the age of component $i$ at epoch $t$,

$t_{i}=\max \left\{t, t+x_{i}^{*}-a g e_{i}\right\}$. 


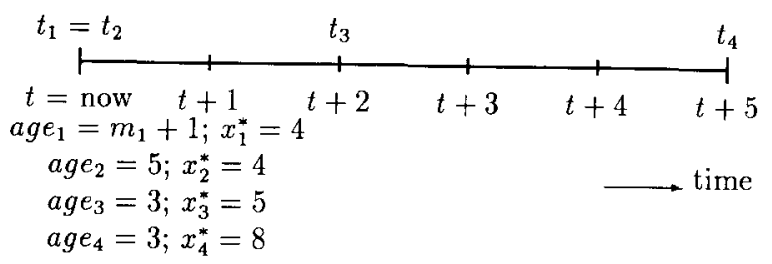

Fig. 1. Example of initial planning.

As $a_{i}$ is defined in Phase 2 as the age of component $i$ at $t_{i}$ in case of no failure, we have that $a_{i} \geqslant x_{i}^{*}$. Only if $t_{i}=t$ and component $i$ has not failed, we have that $a_{i}=a g e_{i}$.

The individual times $t_{i}, i=1, \ldots, n$, induce a finite planning $\left[t, \max _{i} t_{i}\right]$. See Fig. 1 for an example of the $t_{i}$ 's for four components. Suppose that at time $t$ the age $a g e_{i}$ and the individual control limit $x_{i}^{*}$ of component $i, i=1,2,3,4$, are as given in Fig. 1. As component 1 has failed (age ${ }_{1}$ $\left.=m_{1}+1\right)$, it must be replaced immediately, i.e., at epoch $t$. Consequently, $t_{1}=t$ (indeed it holds that $\max \left\{t, t+x_{1}^{*}-a g e_{1}\right\}=\max \left\{t, t+4-m_{1}-\right.$ $1\}=t$ ). The age of component 2 has exceeded its control limit, thus $t_{2}=t$ as well. Under the assumption that component 3 does not fail prematurely, it will reach its control limit at epoch $t+2$, so that $t_{3}=t+2$. Similarly, $t_{4}=t+5$. The corresponding planning horizon equals $\left[t, t_{4}\right]$.

\section{Phase 4: Joint replacement}

Phase 3 provides a finite planning horizon $\left[t, \max _{i} t_{i}\right]$ with an individual planning of the replacement of all $n$ components, based on the state of the system at the current decision epoch $t$. In this phase, this individual planning is considered as an initial plan; the individually planned times can be shifted to enable joint replacement.

In the system we are considering, replacement of component $i$ costs $r_{i}$ (plus $b_{i}$ if it is a corrective replacement). In $r_{i}$ set-up costs $\Delta c$ are included that are the same for all components, which implies that joint replacement of $m$ components yields a cost reduction of $(m-1) \Delta c$. For a combination $C$ of components an optimal replacement time $t_{C}^{*}$ is found by minimising $\sum_{i \in C}$ $h_{i}\left(t_{C^{-}} t_{i}\right)$, the penalty costs of replacing the com- ponents in combination $C$ at time $t_{C}$. Define the savings of combination $C$ as the reduction in set-up costs minus the penalty costs, that is,

$(|C|-1) \Delta c-\sum_{i \in C} h_{i}\left(t_{C}^{*}-t_{i}\right)$.

A combination is cost-effective if its savings are larger than zero.

Phase 4 now aims at determining an optimal combination structure of the replacement of the $n$ components within the planning horizon $\left[t, \max _{i} t_{i}\right]$. An optimal combination structure maximises the total savings (the sum of the savings of all combinations) in the planning horizon. This combination problem can be formulated as a set partitioning problem (see Dekker, Smit and Losekoot [4]), which however can be NP-hard. This is due to the fact that in the worst case all possible combinations of $n$ components, of which there are $2^{n}-1$, have to be investigated. Dekker, Smit and Losekoot [4] present two theorems that can eliminate combinations beforehand, however the effect of these theorems depends heavily on the data, and the remaining problem is often far too large to solve. Wildeman, Dekker and Smit [14] show that under certain general conditions a dynamic programming formulation can be used that can be solved in $\mathscr{O}\left(n^{2}\right)$ time.

They show that if the penalty functions are, for instance, symmetric or congruent (i.e., $\alpha_{i} h_{i}(\cdot)=$ $h_{1}(\cdot)$, for all $i, \alpha_{i}>0$ ), then there exists an optimal combination structure in which the replacements are executed in consecutive order, that is, in the order as initially planned in Phase 3 . This implies that in the worst case only $\frac{1}{2} n(n+1)$ combinations have to be considered. An optimal combination structure can then be found with a dynamic programming algorithm. To apply this algorithm, the components are renumbered such that $t_{1} \leqslant t_{2} \leqslant \cdots \leqslant t_{n}$. (This can be done by standard sorting algorithms that have a time complexity of $\mathscr{O}(n \log n)$.) The algorithm terminates after $n$ iterations, while in each iteration $j$ a best combination with last component $j$ is found. Let the array entry First $[j]$ indicate the first component of this best combination, i.e., if First $[j]=i$, 
then $\{i, \ldots, j\}$ is the best combination found in iteration $j$. Let the total savings of the corresponding optimal combination structure be stored in the array entry TotalSavings $[j]$. Now the dynamic programming algorithm can be outlined as follows.

\section{Initialisation.}

TotalSavings $[0]:=0$.

Iteration 1. The best combination with last component 1 is $\{1\}$, with corresponding optimal combination structure $\{1\}$.

\section{First $[1]:=1$.}

TotalSavings $[1]:=0$.

Iteration $\boldsymbol{j}$. Consider the combinations with last component $j$ in the following order:

$$
\{j\},\{j-1, j\}, \ldots,\{1, \ldots, j\} \text {. }
$$

Find the combination for which the corresponding combination structure covering components $1, \ldots, j$ has largest savings. This is the combination $\{i, \ldots, j\}$ for which TotalSavings $[i-1]+$ savings of $\{i, \ldots, j\}$ is maximal. Next, set

$$
\text { First }[j]:=i \text {. }
$$

$$
\begin{aligned}
\text { TotalSavings }[j]:= & \text { TotalSavings }[i-1] \\
& + \text { savings of }\{i, \ldots, j\} .
\end{aligned}
$$

The best combination structure can be found by backtracking. The corresponding total savings equal TotalSavings[ $n]$.

It is easily shown (see Dekker, Wildeman and Van Egmond [6]) that for identical components the consecutiveness property holds. For nonidentical components more specific information about the (deterioration process of the) components is necessary. When the general conditions are violated, the property of consecutiveness may not hold. In that case the dynamic programming formulation may not be optimal. However, the alternative is to use a set partitioning formulation that can only be heuristically solved for larger number of components. Different set partitioning heuristics can be applied in that case (see, e.g., Wildeman [13]), but it is also possible to use the dynamic programming algorithm as a heuristic. The advantage of this is not only its time complexity, but also its elegant structure and the fact that it can provide an upper bound of the total savings of an optimal combination structure (see Wildeman, Dekker and Smit [14]). We will use the dynamic programming formulation in our approach, even if the consecutiveness property does not hold (but in that case as a heuristic).

\section{Phase 5: Rolling horizon step}

Having applied Phase 4, we now have a combination structure for the $n$ replacements within the planning horizon. However, only the combination (action) planned at the current epoch $t$ is carried out. Components replaced at epoch $t$ will then start with age equal to zero, which may influence the individual planning at future epochs. The same holds for components that fail between epoch $t$ and $t+1$. Consequently, at epoch $t+1$ a new state of the system is considered and the procedure is repeated, starting with Phase 3 (the results of Phases 1 and 2 remain valid).

\subsection{Infinite time horizon}

The computational effort of making a decision at each epoch is only quadratic in the number of components, due to the dynamic programming algorithm. Consequently, many components can be handled, which is not possible when calculating an optimal strategy.

For an optimal strategy the whole Markov chain has to be solved. As each component $i$ has $m_{i}+1$ possible states and there are $n$ components, the state space of the Markov chain consists of $\prod_{i=1}^{n} m_{i}+1$ states, which number grows exponentially in $n$. An optimal strategy for this Markov chain can be found with standard techniques such as successive overrelaxation and policy improvement (see, e.g., Tijms [10]). However, because of the $n$-dimensional state space, this is limited to only a few (about five) components.

For comparison of our approach with an optimal strategy, the average costs have to be calculated. This is done by determining for each possible state in the $n$-dimensional state space an action with our approach, and subsequently calculating the average costs corresponding with the resulting strategy with successive overrelaxation and policy improvement (see also Dekker, Wilde- 
man and Van Egmond [6]). As in the case of calculating an optimal strategy, this is limited to only a small number of components. Problems too big to be solved optimally can be tackled with our approach, however. In that case the average costs have to be determined by simulation.

\subsection{Finite time horizon}

Consider now a finite time horizon $[0, T]$, in which at each decision epoch $0,1, \ldots, T-1$, an action has to be chosen. Epoch $T$ is not a decision epoch, nor the end of the system life; at this epoch a residual value for each component of the system is incurred. For component $i$ being of age $j$ we take as residual value $v_{i}^{j}$, the relative value under the one component control-limit strategy of component $i$ for an infinite time horizon. This corresponds to the situation that after the finite time horizon a new (infinite) time horizon starts, in which the components will be replaced separately. The relative value $v_{i}^{j}$ can then be interpreted as the extra costs of starting this new horizon with component $i$ being of age $j$ instead of being new.

The determination of residual values is rather arbitrary and depends upon future strategies. Choosing a certain structure can have substantial effects, as will be shown later. In the next section we show that the definition given above can yield strange horizon effects. We also show that these effects can be eliminated by taking other residual values.

When our approach is applied in $[0, T]$, an action at an arbitrary epoch $t \in[0, T-1]$ is specified as follows. For each component $i$ an individual replacement time $t_{i}$ is determined, based on the individual control-limit strategy for an infinite time horizon. These times induce a finite planning horizon $\left[t, t_{n}\right]$, which must not exceed $[0, T$ $-1]$. To this end we define $t_{i}=T-1$ as soon as $t_{i} \geqslant T$ for component $i$, by making the penalty function $h_{i}(\cdot)$ infinite for values beyond the time horizon. Thus we have a finite planning horizon $\left[t, \min \left\{t_{n}, T-1\right\}\right]$. Further, the approach is identical as in the case of an infinite horizon: based on the penalty functions a combination structure is found. The combination to be executed at epoch $t$ corresponds with the action to be taken at epoch $t$.

\subsection{What makes our approach heuristic?}

Our approach is based on a simple idea. If today a maintenance activity is due, is it cost-effective to advance execution of other activities that are not yet due so as to have a combined execution today? This is the case if the costs of advancement are less than the cost reduction obtained. A function expressing advancement of a maintenance activity is often convex. This implies that it is perhaps more favourable to execute the combination 'halfway' between the due dates, in which case the activity planned today has to be postponed.

Our approach is a sophisticated elaboration of this notion. The idea of advancement and postponement is expressed in the derivation of the penalty functions. An important step is the determination of future due dates. These are often known with certainty when applying block-replacement strategies. However, when applying age-replacement strategies, as we do in this paper, future execution dates are not known. A first heuristic element of our approach is that most likely execution dates are taken.

A following step is to determine a cost-effective combination structure, rather than only one cost-effective combination. To this end we take a finite planning horizon and find the best combination structure for this planning horizon. A second heuristic element comes in when dynamic programming is applied when the consecutiveness property does not hold.

Another heuristic element of our approach lies in the fact that we firstly assume that shifting execution times (to enable joint execution) is done once only, and that the results are subsequently repeatedly applied on a rolling horizon basis. Our approach is optimal for activities, with known future execution times, that are always executed individually except for a finite horizon in which they may be combined only once. When applying the combination technique in a rolling horizon, we have to answer questions like what finite planning horizon to take and how to incorporate 
harmonisation effects. Harmonisation effects are discussed in the next section.

\section{Computational experiments}

In this section we summarise the results of an investigation into the performance of our approach presented in the previous section. We briefly explain what negative effects play a role and how these effects can be eliminated. Both an infinite and a finite time horizon are considered. A complete description of the numerical experiments is given by Dekker, Wildeman and Van Egmond [6], in an extended report version of this paper.

Comparisons with optimal strategies are only made for a small number of components and states, since only then an optimal strategy can be calculated. Unless stated otherwise, we do not consider more than four components or more than $m_{i}=14$ states per component (excluding the failed state $\left.m_{i}+1\right)$. This makes the examples theoretical, since $m_{i}=14$ is usually a very short maximum lifetime expressed in years or months. However, this disadvantage originates from the problem rather than from our approach: an $m_{i}$ that is too large renders computation time of an optimal strategy unacceptable.

\subsection{Infinite time horizon}

Apart from comparing our approach with an optimal solution (Case 2 below), we first make a comparison with an $(n, N)$-strategy for two identical components.

Table 1

The five lifetime distribution vectors $p_{i}=p^{(1)}, \ldots, p^{(5)}$

$p^{(1)}=(0.80,0.80,0.75,0.66,0.55,0.25,0.15$, $0.10,0.05,0.01)$ $\left(m_{i}=10\right)$

$p^{(2)}=(0.96,0.92,0.87,0.77,0.60,0.40,0.31$, $0.15,0.05,0.05)$

$p^{(3)}=(0.90,0.90,0.88,0.85,0.65,0.45,0.25$, $0.12,0.10,0.10)$

$\left(m_{i}=10\right)$

$\left(m_{i}=10\right)$

$p^{(4)}=(0.81,0.70,0.65,0.61,0.58,0.55,0.53$,

$0.51,0.49,0.48,0.46,0.45,0.44,0.43) \quad\left(m_{i}=14\right)$

$p^{(5)}=(0.99,0.97,0.92,0.84,0.75,0.66,0.56$,

$0.46,0.37,0.29,0.22,0.16,0.11,0.08) \quad\left(m_{i}=14\right)$
Table 2

Percentage increases of $g_{(n, \mathrm{~N})}^{*}$ and $g_{\mathrm{rh}}^{*}$ over $g^{*}$ for the lifetime distribution vector $p^{(5)}$ and the nine combinations of $b_{i}, r_{i}$ and $\Delta c$

\begin{tabular}{lllll}
\hline$b_{i}$ & $r_{i}$ & $\Delta c$ & $(n, N)$ & $r h$ \\
\hline 5 & 1 & 0.4 & 0.00 & 0.00 \\
5 & 2 & 1 & 0.07 & 0.60 \\
5 & 2 & 0 & 0.48 & 0.49 \\
5 & 4 & 3 & 0.00 & 1.33 \\
5 & 4 & 0.5 & 0.12 & 0.35 \\
5 & 7 & 6 & 0.00 & 0.41 \\
5 & 7 & 4 & 0.00 & 0.00 \\
5 & 7 & 1 & 0.03 & 0.26 \\
5 & 12 & 6 & 0.00 & 0.02 \\
\hline
\end{tabular}

\section{Case 1}

Van der Duyn Schouten and Vanneste [11] apply an $(n, N)$-strategy for two identical components 1 and 2 . An $(n, N)$-strategy prescribes to replace a component when it has failed or when its age has reached the value $N$ and, if one of the components is replaced, to replace the other when its age is greater than or equal to $n$. Define the lifetime distribution vector $p_{\mathrm{i}}$ of component $i$ as $p_{i}=\left(p_{i}^{0}, p_{i}^{1}, \ldots, p_{i}^{m_{i}}\right)$.

Van der Duyn Schouten and Vanneste [11] use five lifetime distribution vectors $p_{i}=p^{(1)}, \ldots, p^{(5)}$ and nine combinations of $b_{i}, r_{i}$ and $\Delta c$. This yields a total of 45 different examples, in which $b_{i}$ is constant. Table 1 gives the five lifetime distribution vectors; the nine combinations of $b_{i}, r$ and $\Delta c$ can be found in Table 2. The vectors $p^{(4)}$ and $p^{(5)}$ are obtained as a discretisation of the Weibull $(3,1.4)$ and the Weibull $(6,3)$ distribution, respectively. A Weibull $(\alpha, \beta)$ distributed stochastic variable has a cumulative distribution function

$F(t)=1-\mathrm{e}^{-(t / \alpha)^{\beta}}$.

In Section 2 we assumed that breakdown costs are incurred each time a component fails, whereas in [11] breakdown costs are incurred only once when both components fail simultaneously. To make a good comparison, the evaluation of the costs of our approach (but not the determination of the policy) will here be done according to [11].

Table 2 gives, for the distribution vector $p^{(5)}$ and for the nine combinations of $b_{i}, r_{i}$ and $\Delta c$, 
Table 3

Minimum, average and maximum percentage increases of $g_{(n, N)}^{*}$ and $g_{\mathrm{rh}}^{*}$ over $g^{*}$ averaged over $p^{(1)}, \ldots, p^{(5)}$

\begin{tabular}{lll}
\hline & $(n, N)$ & $r h$ \\
\hline Minimum & 0.00 & 0.00 \\
Average & 0.05 & 0.26 \\
Maximum & 0.51 & 1.33 \\
\hline
\end{tabular}

the percentage increases of $g_{(n, N)}^{*}$ over $g^{*}$, and $g_{\mathrm{rh}}^{*}$ over $g^{*}$. Here $g_{(n, N)}^{*}$ are the average costs of the optimal $(n, N)$-strategy, $g_{\mathrm{rh}}^{*}$ of our rolling horizon approach, and $g^{*}$ of an optimal strategy. The percentage increases of $g_{(n, N)}^{*}$ and $g_{\text {rh }}^{*}$ (over $\left.g^{*}\right)$ are defined as $\left(g_{(n, N)}^{*}-g^{*}\right) / g^{*}$ and $\left(g_{\mathrm{rh}}^{*}-\right.$ $\left.g^{*}\right) / g^{*}$, respectively. In Table 3 the minimum, average and maximum of the percentage increases of $g_{(n, N)}^{*}$ and $g_{\mathrm{rh}}^{*}$ (over $g^{*}$ ) are given, averaged over all five lifetime distribution vectors.

From the results of Table 3 it can be seen that the $(n, N)$-strategy performs very well: the average deviation from an optimal solution is $0.05 \%$. However, our approach performs only slightly worse: $0.26 \%$. The good performance of the $(n, N)$-strategy is probably caused by exploiting the special structure of two identical components. At the same time this is a major drawback of the approach of [11], as only two identical components are dealt with. Based on the embedding technique commonly used for Markov chains, the authors define a Markov chain with a state space that has $\mathscr{O}\left(m_{i}\right)$ instead of $\mathscr{O}\left(m_{i}^{2}\right)$ states (with $m_{i}$ the maximum age of a component). This embedding technique appears to be successful only for systems with a small numbers of components $(\leq 3)$, since it reduces the dimension of the state space only by one.

Our approach does not use the special structure of two identical components, which implies that many more components can be dealt with: taking a decision at a decision epoch requires only $\mathscr{O}\left(n^{2}\right)$ time. Besides, the components need not be identical.

Case 2

To obtain more insight into what effects play a role in the behaviour of our approach when pa-
Table 4

Data of 36 examples

$$
\begin{aligned}
& b_{i}=20 \\
& r_{i}=1,5,10,15,20,25,30,35,40 \\
& \Delta c=10 \%, 35 \%, 60 \% \text { and } 85 \% \text { of } r_{i} \\
& p_{i}=p^{(5)}=(0.99,0.97,0.92,0.84,0.75,0.66,0.56 \\
&\quad 0.46,0.37,0.29,0.22,0.16,0.11,0.08)\left(m_{i}=14\right)
\end{aligned}
$$

rameters change and the number of components grows, we now apply our approach to two, three and four components. We use other parameters than those in [11]. The examples in [11] are somewhat arbitrary, there is no justification for parameters chosen. There are examples with very small control limits $(\approx 2)$ and examples with very large control limits $\left(\approx m_{i}\right)$. These situations are not very likely to appear in practical situations. In our approach breakdown costs are incurred for each failed component. This corresponds more with the practical observation that the probability of simultaneous failure is negligible (under the assumption of independently operating components). For ease of analysis we take identical components, though this is not necessary, of course. However, by doing so, we avoid (large) differences between control limits, which is favourable with respect to discretisation effects.

We take for $r_{i}$ nine different values and, for each of these, four values of $\Delta c$ (that are percentages of $r_{i}$ ). Consequently, we have 36 different examples, in which the breakdown costs $b_{i}$ are constant. The values are chosen such that the ratio of preventive to corrective replacement costs, $r_{i} /\left(r_{i}+b_{i}\right)$, varies from $1 / 21 \approx 0.05$ to $40 / 60 \approx 0.67$. As lifetime distribution vector $p_{i}$ for component $i$ we take the vector $p^{(5)}$ of Table 1. This is the vector that gives the largest deviation of our approach from the optimal solution in

Table 5

Minimum, average and maximum percentage increases of $g_{\mathrm{rh}}{ }^{*}$ over $g^{*}$ for $n=2,3$ and 4 components

\begin{tabular}{lllr}
\hline & $n=2$ & $n=3$ & $n=4$ \\
\hline Minium & 0.00 & 0.00 & 0.02 \\
Average & 0.71 & 2.18 & 4.29 \\
Maximum & 4.70 & 9.92 & 19.00 \\
\hline
\end{tabular}


Table 6

Average savings percentages in case of heuristic replacement or optimal replacement, compared to the 'single control limit strategy" for $n=2,3$ and 4 components

\begin{tabular}{llll}
\hline & $n=2$ & $n=3$ & $n=4$ \\
\hline$\left.g_{\text {scl }}^{*}-g_{\mathrm{rh}}^{*}\right) / g_{\mathrm{scl}}^{*}$ & 11.27 & 13.64 & 13.43 \\
$\left(g_{\mathrm{scl}}^{*}-\mathrm{g}^{*}\right) / g_{\mathrm{scl}}^{*}$ & 11.85 & 15.27 & 16.43 \\
\hline
\end{tabular}

the comparison with the $(n, N)$-strategy of [11] $(1.33 \%$, see Table 2$)$. The data are summarised in Table 4.

In Table 5 the minimum, average and maximum percentage increases of $g_{\mathrm{rh}}^{*}$ over $g^{*}$ are given. The deviation increases with the number of components. This can be explained as follows. A larger number of components imply that there are more opportunities for joint replacement. Moreover, these opportunities are more interesting when set-up costs are high. In the optimal solution of the above examples we indeed see more combining when the number of components and the set-up costs increase, an effect our approach does not take into account, due to the decomposition applied. However, our approach still realises a great part of the savings that can be obtained compared to the implementation of the individual control limits.

This is illustrated in Table 6. When the individual control limits are implemented, we have the situation that joint replacement occurs indirectly when two or more components are at their control limits. Let $g_{\text {scl }}^{*}$ denote the costs of this 'single control-limit strategy'. In Table 6 the average costs of the three policies are compared: In the first row the average savings percentage is shown that can be obtained when applying our approach compared to the single control limit strategy; the second row gives the average savings percentage that can be obtained in case of optimal replacement.

\section{Harmonisation effects}

Our approach relies on a decomposition. As the initial planning in Phase 3 is based on individual control limits, global interactions between components may be ignored. For instance, if there are many components there will frequently be a joint replacement in the optimal solution. This effect may be so strong that in practice a component is hardly ever replaced alone. In that case set-up costs will practically always be shared. To illustrate this effect, consider, for instance, the block replacement of $n$ indentical components. In that case there is a substantial decrease of the optimal replacement interval if $n$ increases (the interval of a single component may even be infinite). If the control limits in our approach are not harmonised (i.e., adjusted to the fact that multiple components are replaced together), this may result in substantial deviations from the optimal solution.

From Table 6 it follows that most of the savings (compared to individual replacement) can be obtained. However, this can be improved considerably when dealing with the harmonisation effect described above. This effect can be dealt with in various ways, which we do not thoroughly scrutinise in this paper. We apply a simple form of harmonisation: in the determination of the individual control limit strategies, we divide the set-up costs by $n$, the number of components, as if set-up costs are always shared (which is often the case in the above examples). Table 7 gives the results of this approach when applied to the 36 examples, for two, three and four components.

From Table 7 we see that the deviation is much smaller, and does not increase significantly with the number of components. In the examples with large set-up costs (viz. $85 \%$ of $r_{i}$ ), the deviation from the optimal solution is mostly $0.00 \%$. The large deviations of $1.94 \%, 2.02 \%$ and $1.88 \%$ are now found in the examples with small control limits (typically 2 and 3 ) and small set-up costs ( $10 \%$ of $r_{i}$ ). This is probably due to the fact that

Table 7

Minimum, average and maximum percentage increases of $g_{\mathrm{rh}}{ }^{*}$ (harmonised) over $g^{*}$ for $n=2,3$ and 4 components

\begin{tabular}{llll}
\hline & $n=2$ & $n=3$ & $n=4$ \\
\hline Minimum & 0.00 & 0.00 & 0.00 \\
Average & 0.14 & 0.17 & 0.21 \\
Maximum & 1.94 & 2.02 & 1.88 \\
\hline
\end{tabular}


the time is not well enough discretised, since in all other examples (even with set-up costs of $10 \%$ ) the deviation stays below $1 \%$. By 'scaling', this discretisation effect may be eliminated, as is suggested by experiments described by Dekker, Wildeman and Van Egmond [6]; the deviation from the optimal solution then stays below one percent.

As one would expect, harmonisation also has a favourable effect on the savings that can be obtained by applying our approach compared to the implementation of the individual control limits; these savings are now quite close to the optimal values.

\subsection{Finite time horizon}

For a finite time horizon we apply our approach also to two, three and four components. We take the same parameters as for an infinite time horizon, see Table 4. As time horizon we take five decision epochs, that is, $T=5$. We firstly took as lifetime distribution vector $p_{i}=p^{(5)}$. This vector yields small control limits in the first examples (especially when $r_{i}=1$ and 5). We calculated for each possible starting state the deviation of the total costs of our approach from the total costs of an optimal solution. As in the case of an infinite time horizon, deviations increase with the number of components.

To make a good investigation into what causes these deviations, discretisation effects because of small control limits have to be avoided. To obtain somewhat larger control limits, we consider other lifetime distribution vectors $p_{i}$, all discretisations of a Weibull $(\alpha, 3)$ distribution, such that control limits vary from 7 to 10 . In Table $8, g_{\mathrm{rh}}^{*}$ equals the total costs of our approach averaged over all possible starting states. Tabulated are the minimum, average and maximum percentage increases of $g_{\mathrm{rh}}^{*}$ over $g^{*}$, taken over all 36 examples. The results do not differ significantly from the results with lifetime distribution vector $p_{i}=$ $p^{(5)}$. The deviations are typically due to a finite horizon effect.

This finite horizon effect is the following. If the individual control limits are relatively small compared to the length of the time horizon, there
Table 8

Minimum, average and maximum percentage increases of $g_{\mathrm{rh}}^{*}$ over $g^{*}$ for $n=2,3$ and 4 components; $p_{i}$ is a discretised Weibull $(\alpha, 3)$ distribution: for $r_{i}=1 \alpha=25$; for $r_{i}=5,10,15$ $\alpha=14 ;$ for $r_{i}=20, \ldots, 40 \alpha=9$

\begin{tabular}{lllr}
\hline & $n=2$ & $n=3$ & $n=4$ \\
\hline Minimum & 0.00 & 0.00 & 0.00 \\
Average & 0.28 & 1.65 & 2.95 \\
Maximum & 1.64 & 7.45 & 14.62 \\
\hline
\end{tabular}

will often be more than one replacement of each component within the horizon. By the definition of residual values (see Section 3.4), it is in general favourable in the optimal solution to replace components in such a way that at the last epoch $T$ - 1 there will be a joint replacement, so as to end with as good as new components. For large set-up costs and many components this may be so important that the optimal strategy will often advance replacements or plan extra replacements to make this possible. Our approach does not take this into account, as it does not use the residual values. The effect typically occurs for starting states with large differences between ages.

\section{Eliminating the horizon effect}

The finite time horizon effect causes a very capricious character of the optimal strategies, whereas our approach has a more stable behaviour. The finite horizon effect is eliminated in three cases, viz. when: 1) The individual control limits are relatively large compared to the length of the time horizon; 2) a residual value is chosen that corresponds to the situation that joint replacement is also possible after the finite time horizon; and 3) the ages are not very different.

In case 1) the finite horizon effect is not so strong, because it is not very favourable in an optimal solution to have more than one replacement within the horizon. This can for instance be seen in an example with two components. In Table 9 we see the influence of the lifetime distribution on the deviation for different lengths $T$ of the time horizon. For $\alpha=21$ the deviation for a time horizon of length 5 is very small. This is a scaling effect: Deviations grow again for 
larger horizons. Thus the finite horizon effect does not disappear; only if the horizon is small enough, the effect falls beyond the horizon. However, this observation is interesting, since in practice the control limits are often relatively large compared to the length of the time horizon.

Case 2) and case 3) apply when the multi-component system is maintained on a rolling horizon basis. In practical situations a finite time horizon is mostly used in a rolling horizon approach; the actions in the first period(s) of the horizon are implemented, after which a new finite time horizon starts, and so on. In that case we should define residual values that correspond to the situation that after the finite horizon components can still be jointly replaced. We now take as residual values those of our approach for an infinite time horizon. This does not change the solution according to our approach, since we do not use the residual values for the determination of a strategy; it does, however, change the average costs and, consequently, the optimal solution. The optimal strategies become more stable because they are not any longer very eager to end with new components.

Another very important observation is that in a rolling horizon approach not all starting states are equally probable; in a long-term plan, ages of components will be more synchronised. That is why we also consider the deviation of our approach from an optimal strategy when the deviations per starting state are weighted according to the limit probabilities in the infinite horizon case. These are the steady-state probabilities of being in a state $s$ of the $n$-dimensional state space. We took the probabilities of the strategy induced by our approach, for an infinite time horizon. To see what happens when this rolling horizon view is

Table 9

Percentage increases of $g_{\mathrm{rh}}^{*}$ over $g^{*}$ for two components and different values of $T ; r_{i}=30, \Delta c=25.5\left(=85 \%\right.$ of $\left.r_{i}\right), p_{i}$ is a discretised Weibull $(\alpha, 3)$ distribution

\begin{tabular}{rllllllllll}
\hline$\alpha$ & $T$ & 10 & & & & & & & & \\
\cline { 2 - 10 } & 1 & 2 & 3 & 4 & 5 & 6 & 7 & 8 & 9 & 10 \\
\hline 6 & 0.00 & 0.01 & 0.30 & 2.01 & 2.22 & 1.36 & 0.85 & 0.98 & 2.38 & 2.05 \\
9 & 0.00 & 0.00 & 0.01 & 0.05 & 1.03 & 1.96 & 1.72 & 1.20 & 0.81 & 0.57 \\
21 & 0.00 & 0.00 & 0.00 & 0.01 & 0.02 & 0.05 & 0.11 & 0.20 & 0.58 & 1.24
\end{tabular}

Table 10

Percentage increases of $g_{\mathrm{rh}}^{*}$ over $g^{*}$ for the original and rolling horizon definition; $p_{i}$ is a discretised Weibull $(\alpha, 3)$ distribution

\begin{tabular}{|c|c|c|c|c|}
\hline & \multicolumn{2}{|c|}{$\begin{array}{l}r_{i}=5 \\
\Delta c=4.25 \\
\left(=85 \% \text { of } r_{i}\right) \\
\alpha=14\end{array}$} & \multicolumn{2}{|c|}{$\begin{array}{l}r_{i}=30 \\
\Delta c=25.5 \\
\left(=85 \% \text { of } r_{i}\right), \\
\alpha=9\end{array}$} \\
\hline & $n=2$ & $n=3$ & $n=2$ & $n=3$ \\
\hline Original definition & 1.07 & 2.22 & 1.03 & 5.40 \\
\hline Rolling horizon definition & 0.00 & 0.00 & 0.00 & 0.17 \\
\hline
\end{tabular}

applied, we arbitrarily take two combinations of $r_{\mathrm{i}}$ and $\Delta c$, both for two and three components, and with $p_{\mathrm{i}}$ according to a discretised Weibull $(\alpha, 3)$ distribution (these are four of the examples summarised in Table 8 ).

In Table 10 the results are tabulated. The 'Rolling horizon definition' corresponds to the alternative definition of residual values and the deviations weighted according to the limit probabilities as described above. The solutions according to this definition are compared to the solutions according to the original definition of the residual values and unweighted deviation. It is clear that our approach performs very well when the residual values according to the rolling horizon definition are used.

\section{Conclusions}

In this paper we analysed a multi-component replacement problem in an operational planning phase. Given an infinite or a finite time horizon with discrete decision epochs, the objective was to specify a replacement action at each epoch, so as to minimise average or total costs. We presented a rolling horizon approach based on a component decomposition and we used Markov decision theory and dynamic programming. $\mathrm{Nu}$ merical experiments for a maximum of four components show that strategies induced by our approach yield average costs less than one percent above the optimal values (after dealing with harmonisation and horizon effects).

The advantage of our approach is that an action in a certain state is determined indepen- 
dently of other states. Since the computational effort of determining an action is only quadratic in the number of components, this implies that we can handle many more components than other approaches published so far. Most of these approaches are limited to a few (not more than five) components. Unfortunately, this also implies that a comparison with other approaches for many components is difficult.

Our approach has some interesting potentials that are not scrutinised in this paper and that can be subjects for future research. Since maintenance activities are combined on a short-term basis, typical operational circumstances such as one-off savings can be taken into account; it is interesting to study the applicability of the approach when dealing with other operational circumstances such as manpower constraints. Our approach also provides planning possibilities, which is an important feature when work preparation is necessary. Another aspect is that insight is obtained into why maintenance activities are combined. The penalty functions indicate how much it costs to execute activities at times other than initially planned. As new maintenance decisions are generated quickly, this yields possibilities for interactive planning. Our approach is flexible in the sense that it can be applied to many maintenance systems. In this paper lifetime is used as state parameter. Our approach may also be applied to, for instance, a system in which the state of deterioration can only be determined through inspection and repair is necessary to renew a component. In such a system, decisions concerning when to inspect and when to repair have to taken.

\section{References}

[1] Bäckert, W., and Rippin D.W.T., "The determination of maintenance strategies for plants subject to breakdown," Computers and Chemical Engineering 9 (1985) 113-126.
[2] Cho, D.I., and Parlar, M., "A survey of maintenance models for multi-unit systems," European Journal of Operational Research 51 (1991) 1-23.

[3] Dekker, R., and Roelvink, I.F.K., "Marginal cost criteria for preventive replacement of a group of components," European Journal of Operational Research 84 (1995) 467480.

[4] Dekker, R., Smit, A.C.J.M., and Losekoot, J.A., "Combining maintenance activities in an operational planning phase: A set-partitioning approach," IMA Journal of Mathematics Applied in Business and Industry 3/4 (1992) 315-331.

[5] Dekker, R., and Wildeman, R.E., "Combining activities in an operational planning phase: An application to joint replenishment," Technical Report 9250/A, Econometric Institute, Erasmus University Rotterdam, 1992.

[6] Dekker, R., Wildeman, R.E., and Van Egmond, R., “Joint replacement in an operational planning phase-extended version," Technical Report 9362/A, Econometric Institute, Erasmus University Rotterdam, 1993.

[7] Geurts, J.H.J., "Optimal age replacement versus condition based replacement: Some theoretical and practical considerations," Journal of Quality Technology 15/4 (1983) 171-179.

[8] Haurie, A., and L'Ecuyer, P., "A stochastic control approach to group preventive replacement in a multicomponent system," IEEE Transactions on Automatic Control 27 (1982) 387-393.

[9] Howard, R.A., Dynamic Programming and Markov Processes, Wiley, New York, 1960.

[10] Tijms, H.C., Stochastic Modelling and Analysis, Wiley, New York, 1986.

[11] Van der Duyn Schouten, F.A., and Vanneste, S.G. "Analysis and computation of ( $n-N)$-strategies for maintenance of a two-component system," European Journal of Operational Research $48 / 2$ (1990) 260-274.

[12] Wijnmalen, D.J.D., and Hontelez, J.A.M., "Opportunistic condition-based repair and inspection strategies for components of multi-component maintenance system with discounts," Paper presented at the EURO XII/TIMS XXXI Conference in Helsinki (submitted for publication in European Journal of Operational Research), 1992.

[13] Wildeman, R.E., "Combined maintenance scheduling," Master's thesis, Leiden University (unpublished), 1991.

[14] Wildeman, R.E., Dekker, R., and Smit, A.C.J.M., "A dynamic policy for grouping maintenance activities," Technical Report 95371A, Economic Institute, Erasmus University, Rotterdam, 1995.

[15] Worm, J.M., and Van Harten, A., "Model based decision support for planning of road maintenance," to appear in Reliability Engineering \& Systems Safety. 